\title{
A prospective study of Trichomonas vaginalis and prostate cancer risk among African American men
}

\author{
Jay H. Fowke ${ }^{1,4^{*}}$, Xijing Han², J. F. Alderete ${ }^{3}$, Kelvin A. Moses ${ }^{4}$, Lisa B. Signorello ${ }^{5}$ and William J. Blot ${ }^{1,2}$
}

\begin{abstract}
Background: African Americans (AA) have a higher prevalence of Trichomonas vaginalis (Tv) infection and a higher prostate (PC) risk. Past studies suggest an association between TV seropositivity and PC, and therefore we prospectively investigated this association among AA men.

Results: Incident PC cases were individually matched to controls in a nested case-control study within the Southern Community Cohort Study (SCCS). Primary analysis included 296 PC cases and 497 race-matched controls. Levels of TV antibody response were measured by ELISA in serum collected at baseline. TV antibody response did not significantly differ between cases and controls overall or within AA participants (253 AA cases). There were no significant associations or trends between levels of $T v$ response and PC risk or the diagnosis of aggressive PC.
\end{abstract}

Conclusion: We found no evidence of a prospective association between baseline TV infection and PC risk in AA men. Tv infection in men may have substantial health implications in HIV transmission and reproductive outcomes, but may not impact future PC risk in AA men at high-risk for PC. Further efforts need to define past vs. present TV infection and to separate pathophysiology from PC detection.

Keywords: Prostate cancer, Trichomonas vaginalis, Race

\section{Background}

Trichomonas vaginalis (Tv) is a sexually transmitted protozoan parasite. In men, urethral infection may ascend to the prostatic urethra and glandular tissue. With asymptomatic infection common, $T v$ exposure may induce a sustained inflammatory response to advance prostate carcinogenesis [1]. Analysis of the Health Professionals Follow-up Study and the Physicians Health Study found that $T v$ antibody seropositivity was significantly associated with prostate cancer (PC) risk [2,3] or PC death [2]. In contrast, $T v$ seropositivity was not associated with PC risk in the Prostate Cancer Prevention Trial (PCPT) [4] or a population-based case-control analysis [5]. The purpose of this study was to determine the prospective

\footnotetext{
*Correspondence: Jay.fowke@vanderbilt.edu

${ }^{1}$ Departments of Medicine, Institute of Medicine and Public Health, Vanderbilt University Medical Center, 2525 West End Ave, 12th floor, Nashville, TN 37232, USA

Full list of author information is available at the end of the article
}

relationship between $T v$ infection and PC risk among African American (AA) men. Past studies included few AA men, although there are data suggesting the prevalence of $T v$ infection and the risk of PC may be greater than among white men [6].

\section{Methods}

Details of the Southern Community Cohort Study (SCCS) have been published [7]. Incident PC cases among nearly 35,000 male participants enrolled at age 40-79 during 2002-2009 were identified through linkages through 2013 with state tumor registries and the National Death Index. All participants provided written informed consent, and all protocols were approved by Institutional Review Boards at Vanderbilt University and Meharry Medical College. All SCCS data can be requested through an online request (southerncommunitystudy.org). The request will be reviewed by the SCCS Data and Biospecimen Use Committee to ensure that it is 
scientifically justified and that participant confidentiality is preserved. Two controls per case were selected by incidence density sampling and were individually matched by age ( 5 years), race (self-reported black or white), site, and time of donation of blood samples at study entry. A blood sample was collected at baseline recruitment, and serum was frozen-stored at $-80^{\circ} \mathrm{C}$. Serum was assayed in duplicate for antibodies against $T v$ by ELISA to detect the IgG antibodies against the purified recombinant $T v$ $\alpha$-actinin protein and assessed spectrophotometrically [8]. We previously assigned scores from 0 to $4+$ to serum based on the calculation of $\mathrm{P} / \mathrm{N}$ values obtained using the protein ACT-P2 as the target [2-4, 8]. For this study, cutoff points for seropositivity were obtained by dividing the average $\mathrm{OD}_{405 \mathrm{~nm}}$ of the seropositive control serum by the corresponding seronegative control serum. The lowest seronegative control was assigned a score of 0 (zero). Values from 0 to the next lowest was given a score of $1+$, and subsequent scores of $2+, 3+$, and $4+$ were assigned similarly as values increased. The sera with $\mathrm{P} / \mathrm{N}$ scores $\leq 2$ were negative as evidenced by lack of detection of any T. vaginalis proteins by immunoblot [8]. Scores $\geq 3$ were positive and had antibody to $\alpha$-actinin and other trichomonad proteins [8]. We used conditional logistic regression to compute odds ratios (OR) and $95 \%$ confidence intervals while controlling for household income. Cancer stage or Gleason score from state tumor registries providing these data were also analyzed after controlling for age, race, and income.

\section{Results}

The majority of the study population was over 60 years of age at recruitment $(\mathrm{n}=549,62 \%)$, and reported a household income less than $\$ 15,000 /$ year $(\mathrm{n}=512,58 \%)$.
Table 1 Trichomonas vaginalis and association with prostate cancer risk

\begin{tabular}{lllll}
\hline T. vaginalis & Cases & Controls & OR & 95 \% Cl \\
\hline $\mathrm{n}$ & 296 & 585 & & \\
Mean (SD) & $0.26(0.11)$ & $0.26(0.11)$ & & \\
Serostatus & & & & \\
$\quad$ Negative & $227(76.7 \%)$ & $461(78.8 \%)$ & 1.0 & Ref \\
Positive & $69(23.3 \%)$ & $124(21.2 \%)$ & 1.11 & $0.77-1.61$ \\
Score & & & & \\
$\quad 0$ & $60(20.3 \%)$ & $132(22.6 \%)$ & 1.0 & Ref \\
1 & $95(32.1 \%)$ & $168(28.7 \%)$ & 1.18 & $0.77,1.82$ \\
2 & $72(24.3 \%)$ & $161(27.5 \%)$ & 0.94 & $0.58-1.53$ \\
3 & $50(16.9 \%)$ & $84(14.4 \%)$ & 1.25 & $0.74-2.11$ \\
$4+$ & $19(6.4 \%)$ & $40(6.8 \%)$ & 0.98 & $0.48-1.97$ \\
African Americans Only & & & \\
$\mathrm{n}$ & 253 & 497 & & \\
Mean (SD) & $0.26(0.11)$ & $0.26(0.11)$ & & \\
Serostatus & & & & \\
Neg & $191(75.5 \%)$ & $386(77.7 \%)$ & 1.0 & Ref \\
Pos & $62(24.5 \%)$ & $111(22.3 \%)$ & 1.12 & $0.76-1.66$ \\
Score & & & & \\
0 & $50(19.8 \%)$ & $104(20.9 \%)$ & 1.0 & Ref \\
1 & $80(31.6 \%)$ & $145(29.2 \%)$ & 1.10 & $0.69-1.76$ \\
2 & $61(24.1 \%)$ & $137(27.6 \%)$ & 0.87 & $0.51-1.47$ \\
3 & $44(17.4 \%)$ & $74(14.9 \%)$ & 1.18 & $0.67-2.08$ \\
$4+$ & $18(7.1 \%)$ & $37(7.4 \%)$ & 0.95 & $0.45-1.97$ \\
\hline Adjuted & & & &
\end{tabular}

Adjusted for age at diagnosis, race, income

a Adjusted for age at diagnosis and income*

Approximately $85 \%$ of the study population was AA. Table 1 summarizes the association between $T v$ seropositive status and PC. Mean antibody response levels were

Table 2 Trichomonas vaginalis seropositive status and diagnosis of aggressive prostate cancer

\begin{tabular}{|c|c|c|c|c|}
\hline \multicolumn{5}{|c|}{ Diagnosis of aggressive $P C^{a}$} \\
\hline & Gleason $<7$ & Gleason $\geq 7$ & OR & $95 \% \mathrm{Cl}$ \\
\hline$n$ & 74 & 101 & & \\
\hline Mean (SD) & $0.25(0.10)$ & $0.26(0.10)$ & & \\
\hline \multicolumn{5}{|l|}{ Serostatus } \\
\hline Negative & $60(81.1 \%)$ & $80(79.2 \%)$ & & \\
\hline \multirow[t]{2}{*}{ Positive } & $14(18.9 \%)$ & $21(20.8 \%)$ & 1.04 & $0.48-2.26$ \\
\hline & Stage $0-1$ & Stage 2-4 & & \\
\hline$n$ & 230 & 40 & & \\
\hline Mean (SD) & $0.25(0.12)$ & $0.26(0.10)$ & & \\
\hline \multicolumn{5}{|l|}{ Serostatus } \\
\hline Negative & $179(77.8 \%)$ & $30(75.0 \%)$ & & \\
\hline Positive & $51(22.2 \%)$ & $10(25.0 \%)$ & 1.23 & $0.55-2.74$ \\
\hline
\end{tabular}

Stage and grade data were not provided by every tumor registry

a Adjusted for age at diagnosis, race, and income 
similar between cases and controls (all $p>0.05$ ). There was no significant association between $T v$ and PC in the total study population, or when restricting to AA men. Furthermore, $T v$ exposure was not associated with PC Gleason score of 7 or more, or stage 2-4 PC at diagnosis, in a case-only analysis (Table 2).

\section{Discussion}

Tv disproportionately affects minorities and lowincome populations [6]. However, unlike two past studies of predominately white professionals $[2,3]$, we found no evidence of association within AA men. A post hoc power calculation based on a similar matched nested case-control design and assuming a $25 \%$ exposure, Type I error of $5 \%$, and $80 \%$ power, would be able to detect an OR $=1.42$, suggesting a sufficient sample size to identify a moderate association. Residual confounding is unlikely to have led to a null association. As previously detailed, differences in patient characteristics and detection protocols could explain differences in results across studies [4]. Similarly, an inflammatory effect from Tv would likely increase PSA levels, and thus increase the likelihood of detecting an asymptomatic PC and lead to a positive association. No association between Tv seropositivity and PC was found in the PCPT, with rigorous control for $\mathrm{PC}$ screening including an end of study prostate biopsy [4]. Alternatively, analysis of the Prostate, Lung, Colorectal and Ovarian cancer screening trial found $T v$ seropositivity significantly associated with benign prostatic hyperplasia [9], suggesting $T v$ may exacerbate lower urinary tract symptom severity and possibly lead to a PC detection. [10] Tv seropositivity is relatively stable over time [10], limiting the ability to identify an etiologically relevant time of exposure. Our prospective analysis found no association between baseline Tv seropositivity and future PC risk, and further efforts are needed to define past vs. recent $T v$ infection and to separate pathophysiology from the detection of PC.

\footnotetext{
Abbreviations

AA: African American; Cl: confidence interval; OR: odds ratio; PC: prostate cancer; PCPT: prostate cancer prevention trial; P/N: positive/negative ratio; SCCS: Southern Community Cohort Study; Tv: Trichomonas vaginalis.
}

\section{Authors' contributions}

Conception and design (WJB, LBS), laboratory analysis (JFA), statistical analysis (XJ, JHF, WJB), primary author (JHF), contributing authors involved in interpretation and drafting manuscript (WJB, KAM, JFA). All authors read and approved the final manuscript.

\section{Author details}

${ }^{1}$ Departments of Medicine, Institute of Medicine and Public Health, Vanderbilt University Medical Center, 2525 West End Ave, 12th floor, Nashville, TN 37232, USA. ${ }^{2}$ International Epidemiology Institute, Rockville, MD, USA. ${ }^{3}$ School of Molecular Biosciences, Washington State University, Pullman, WA, USA. ${ }^{4}$ Department of Urologic Surgery, Vanderbilt University Medical Center, Nashville, USA. ${ }^{5}$ Division of Cancer Prevention, National Cancer Institute, National Institutes of Health, Bethesda, MD, USA.

\section{Acknowledgements}

This work was supported by NIH RO1CA92447.

\section{Competing interests}

The authors declare that they have no competing interests.

\section{Declarations}

All research protocols were approved by IRBs at Vanderbilt University (IRB00000475) and Meharry Medical College (IRB00000529), and all participants provided written informed consent.

Received: 11 January 2016 Accepted: 8 April 2016

Published online: 18 April 2016

\section{References}

1. Chen Y, Huang Y, Platz E, Alderete J, Zheng L, Rider J, Kraft P, Giovannucci E, Sutcliffe S. Prospective study of effect modification by Toll-like receptor 4 variation on the association between Trichomonas vaginalis serostatus and prostate cancer. Cancer Causes Control. 2013;24(1):175-80.

2. Stark JR, Judson G, Alderete JF, Mundodi V, Kucknoor AS, Giovannucci EL, Platz EA, Sutcliffe S, Fall K, Kurth T, et al. Prospective study of Trichomonas vaginalis infection and prostate cancer incidence and mortality: physicians' health study. J Natl Cancer Inst. 2009;101(20):1406-11.

3. Sutcliffe S, Giovannucci E, Alderete JF, Chang T-H, Gaydos CA, Zenilman JM, De Marzo AM, Willett WC, Platz EA. Plasma antibodies against Trichomonas vaginalis and subsequent risk of prostate cancer. Cancer Epidemiol Biomarkers Prev. 2006;15(5):939-45.

4. Sutcliffe S, Alderete JF, Till C, Goodman PJ, Hsing AW, Zenilman JM, De Marzo AM, Platz EA. Trichomonosis and subsequent risk of prostate cancer in the prostate cancer prevention trial. Int J Cancer. 2009;124(9):2082-7.

5. Shui IM, Kolb S, Hanson C, Sutcliffe S, Rider JR, Stanford JL: Trichomonas vaginalis infection and risk of advanced prostate cancer. Prostate. 2016.

6. Miller WC, Swygard H, Hobbs MM, Ford CA, Handcock MS, Morris M, Schmitz JL, Cohen MS, Harris KM, Udry JR. The prevalence of Trichomoniasis in young adults in the United States. Sex Transm Dis. 2005;32(10):593-8.

7. Signorello LB, Hargreaves MK, Steinwandel MD, Zheng W, Cai Q, Schlundt DG, Buchowski MS, Arnold CW, McLaughlin JK, Blot WJ. Southern community cohort study: establishing a cohort to investigate health disparities. J Natl Med Assoc. 2005;97(7):972-9.

8. Neace CJ, Alderete JF. Epitopes of the highly immunogenic Trichomonas vaginalis alpha-actinin are serodiagnostic targets for both women and men. J Clin Microbiol. 2013;51 (8):2483-90.

9. Breyer BN, Huang W-Y, Rabkin CS, Alderete JF, Pakpahan R, Beason TS, Kenfield SA, Mabie J, Ragard L, Wolin KY, et al. Sexually transmitted infections, benign prostatic hyperplasia and lower urinary tract symptomrelated outcomes: results from the prostate, lung, colorectal and ovarian cancer screening trial. BJU Int. 2016;117(1):145-54.

10. Sutcliffe S, Alderete JF, Neace C, Joyce PA, Gaydos CA, Huth JIA, Mucci LA, Signorello LB. Persistence of Trichomonas vaginalis serostatus in men over time. Cancer Cause Control. 2015;26(10):1461-6. 